\title{
Mm-Wave STSK-aided Single Carrier Block Transmission for Broadband Networking
}

\author{
Talha Faizur Rahman and Aamir Habib \\ Dept. of Electrical Engineering \\ Institute of Space Technology \\ Islamabad, Pakistan \\ Email: talha.rahman@alumni.unitn.it, \\ aamir.habib@ist.edu.pk
}

\author{
Claudio Sacchi \\ Dept. of Information Engineering \\ and Computer Science (DISI) \\ University of Trento \\ Trento, Italy \\ Email: claudio.sacchi@unitn.it
}

\author{
Mohammed El-Hajjar \\ Dept. of Electronics and \\ Computer Science \\ University of Southampton \\ Southampton, UK \\ Email: meh@ecs.soton.ac.uk
}

\begin{abstract}
Millimeter wave (mm-Wave) communications has been considered as a strong candidate for future wireless standards, due to the large available bandwidth in the order of gigahertz. As a result, a plethora of future services and application-oriented scenarios can be conceived, under suitable propagation conditions. Accurate physical (PHY) layer design plays a vital role in the deployment of robust transmission systems able to efficiently exploit the large bandwidth portions available in the mm-Wave frequencies. In this paper, we propose Space-Time Shift Keying (STSK) MIMO coding combined with Cyclic-Prefixed Single Carrier (CP-SC) block transmission for broadband data exchange over frequency-selective mm-Wave channels. STSK allows to exploit transmit and receive diversity with better performance when compared with state-of-the-art MIMO techniques only relying on receive diversity, such as, for example Spatial Modulation (SM). The performance of CP-SCSTSK and CP-SC-SM have been assessed in the presence of phase noise and imperfect channel estimation, considering 2 and 4 elements MIMO systems and LoS indoor vs. nLoS outdoor $73 \mathrm{GHz}$ multipath channels. We show that the STSK-based solution using MMSE Frequency-Domain Equalization (FDE) is very robust against the aforementioned impairments and clearly outperforms CP-SC-SM at the price of a slight increase of receiver complexity and a throughput reduction of $50 \%$ when $4 \times 4$ MIMO systems are considered.
\end{abstract}

Index Terms-Single Carrier, Space Time Shift Keying, Millimeter Wave, Imperfect Channel Estimation, Phase Noise, Eband.

\section{INTRODUCTION}

Due to the proliferation of smart handheld devices with applications and services that require seamless broadband connectivity on the go, the demand of high data rate is rapidly increasing. For that reason, researchers are looking to explore new frequency bands in order to meet future communication requirements. Recently lots of research emphasis has been drawn on using the millimeter wave (mm-Wave) frequency bands, especially in the E-band $(71-76 \mathrm{GHz}$ and $81-86 \mathrm{GHz})$, where a total amount of bandwidth equal to $10 \mathrm{GHz}$ is available.

Coldrey et.al. in [1] proposed mm-Wave solution for nonLine-of-Sight (nLoS) applications where it is claimed that Quality of Service (QoS) increases by pointing receiver antenna to the direction of best approaching reflected ray. $73 \mathrm{GHz}$ band has shown better atmospheric absorption characteristics compared to the $60 \mathrm{GHz}$ frequency band for wideband connections [2]. Based on these analyses, the authors in [2] considered advanced techniques, like MIMO and beamforming, to show the feasibility of $\mathrm{mm}$-Wave $73 \mathrm{GHz}$ communication in both LoS and nLoS environments.

IEEE 802.11ad standard has recently approved both Single Carrier block transmission with Frequency Domain Equalization (SC-FDE) and OFDM together with single stream MIMO for multigigabit WLANs using $60 \mathrm{GHz}$ band [3]. For shorter distances where LoS is often guaranteed, OFDM is a viable transmission solution. However, due to their multicarrier nature, OFDM systems are vulnerable to nonlinear distortions and phase noise. On the other hand SC-FDE, characterized by lower Peak-to-Average Power Ratio (PAPR), has performance comparable to that of OFDM, without significant increase in complexity [4]. For these reasons, SC in combination with MIMO signal processing is being regarded as a possible candidate for future-generations wireless standards.

In [5], authors considered the hardware configuration and Integrated Circuit (IC) package development for multi-level single carrier working at $\mathrm{mm}$-Wave with the aim is to achieve $1 \mathrm{Gbps}$ with inexpensive equipement. Single carrier with Iterative Block Frequency Domain Equalization (IB-FDE) is proposed in [6] where MIMO SC-IB-FDE shows significant resilience against imperfect channel estimation for high speed mobility using lower order of modulation. As far SC-FDE is considered in combination with low-complexity MIMO coding techniques, Spatial modulation-aided [7] zero-padded single carrier technique (ZP-SC-SM) is proposed in [8] for dispersive channels with low-complexity partial interference cancellation receiver that achieves full multipath and receive diversity as compared to CP-SC in SM-MIMO systems. ZP-SC system save transmission energy, however it requires complex timefrequency synchronization which makes its practical implementation a challenge. On the other hand, SM requires to have high speed switching in analog domain while operating in millimeter frequency band, thus increasing the system cost or even reduces the full operability of transmitter for high-data rate applications.

In this paper, Cyclic Prefixed (CP) single-carrier block modulation is proposed in conjunction with Space-Time Shift 
Keying (STSK) MIMO coded transmission in order to improve link robustness in mm-Wave broadband networking applications. STSK has been introduced by Sugiura, Chen and Hanzo in [9] where authors state that the proposed technique is capable of providing useful degrees of freedom in order to efficiently manage the tradeoff between diversity and multiplexing. In [9], OFDM transmission is considered in combination with STSK in order to exploit the lowcomplexity maximum-likelihood detection (ML), performed per subcarrier. Due to the aforesaid issues related to nonlinear distortion susceptibility, the adoption of STSK-OFDM may be hindered in low-power systems, like indoor WLANs, and might be problematic also in small-cell outdoor transmission systems characterized by hardly-constrained link budget. The perspective looks more favourable for the use of STSKOFDM in other kind of applications, for instance in the mm-wave wireless backhaul, as shown by Sacchi et al. in [10]. Unlike MIMO-OFDM, the ML complexity is inevitably high in wideband MIMO single carrier systems for increased number of taps and block size [8] [11]. Thus SC SM/STSK systems must resort to low-complexity near-optimum FDE as proposed in [11]. This last solution, based on MMSE-FDE, has been considered in the present paper in order to achieve nearoptimum performance with tolerable computational burden.

To the best of the authors' knowledge, cyclic prefixed SC with STSK has not been analysed in literature. In [12], Linear Dispersion Codes (LDC) are tested with CP-SC systems. The high rate $\mathrm{LDC}$ harvest time-diversity together with $\mathrm{CP}-\mathrm{SC}$ that results in performance improvement over single-antenna $\mathrm{CP}$ $\mathrm{SC}$ and LDC-CP-OFDM. Our approach is different from [12] and is based on STSK principle.

As SC waveform is being considered with single-user MIMO for short range IEEE 802.11ad indoor systems, we take a further step ahead by proposing CP-SC-STSK scheme as a viable PHY-layer solution for both short range indoor mmWave links, usually characterized by LoS propagation (indoor scenario) and longer range outdoor communication links that work in nLoS conditions [2] (outdoor scenario). The novelty of our work is two fold:

- We propose STSK-aided Cyclic Prefixed Single Carrier (CP-SC-STSK) for millimeter wave wideband applications in LoS and nLoS dispersive channels;

- We consider convolutional coding (i.e. 1/2 code rate) in CP-SC-STSK against practical challenges, like phase noise and imperfect channel estimation and compared performance with state-of-the-art CP-SC-SM technique.

The paper is structured as follows: section II includes a comparative description of the proposed CP-SC-STSK transmission system along with the state-of-the-art solution based on CP-SC-SM. Section III will highlight the mm-wave channel modelling and the impact of phase noise and nonideal channel estimation on CP-SC-STSK and CP-SM transmission. Section IV will discuss selected simulation results concerning Eband transmission in indoor LoS and outdoor nLoS scenarios. Finally, conclusion will be drawn in Section V.

\section{CP-SC MIMO TRANSMISSION SYSTEM DESCRIPTION}

A. The proposed STSK-aided Cyclic-Prefixed Single-Carrier Block Transmission (CP-SC-STSK)

In this paper, we consider mm-Wave transmission in a scenario where $M$ transmit antennas are sending information to $N$ receive antennas. The block diagram of the proposed CP-SC-STSK transmission system is shown on the upper side of Fig. 1. The channel-encoded bit-stream is further encoded by the STSK space-time mapping algorithm. Then, the generated MIMO-STSK codeword is stored into a buffer of size $B$ bits and the resulting block is transmitted by $M$ cyclic-prefixed single-carrier transmitters, employing bandlimited pulse shaping filters. Finally the CP-SC-STSK signal is D/A converted, frequency-upconverted and transmitted over the wireless channel.

The STSK codeword generation is $\log _{2}(Q)$ bits index a dispersion matrix $q$, among $Q$ available dispersion matrices of size $M \times T, T \leq M$ being the number of columns of the dispersion matrix. The selected dispersion matrix disperses the energy of input information symbol, taken from a $K$-ary PSK or QAM constellation, which is indexed by the remaining bits of the transmitted binary string. The transmitted STSK
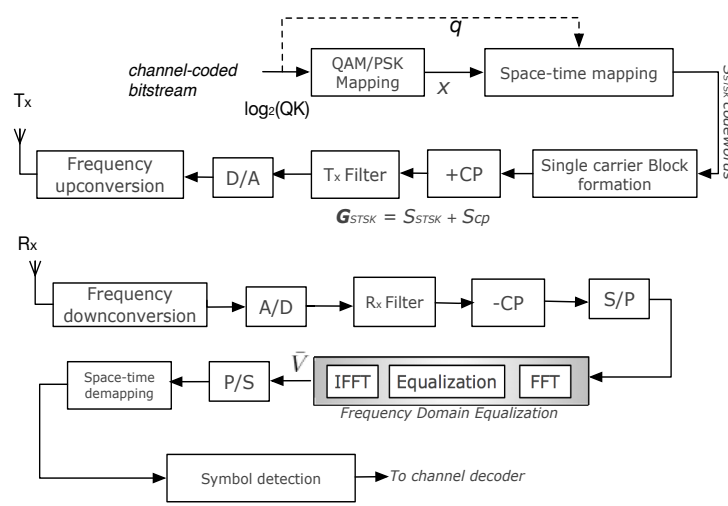

Fig. 1. Block diagram of the proposed Space-Time Shift Keying (STSK)-aided Cyclic-Prefixed Single-Carrier Transceiver System (CP-SC-STSK).

codeword can be expressed as follows:

$$
\begin{array}{r}
\mathbf{X}_{\mathrm{STSK}}=\mathbf{0}_{\mathbf{M} \times \mathbf{T}}+\cdots+S_{k} \times \mathbf{A}_{\mathbf{q}}+ \\
\cdots+\mathbf{0}_{\mathbf{M} \times \mathbf{T}}
\end{array}
$$

where: $S_{k}$ is the complex information symbol and $\mathbf{A}_{\mathbf{q}}$ is the selected dispersion matrix, whose generic form can be represented as:

$$
\mathbf{A}_{\mathbf{q}}=\left[\begin{array}{ccc}
a_{1}^{1} & \cdots & a_{1}^{T} \\
\vdots & \ddots & \vdots \\
a_{M}^{1} & \cdots & a_{M}^{T}
\end{array}\right] \in \mathbb{C}^{M \times T}
$$

The zero matrices in (1) are related to the de-activated dispersion matrices (only one matrix, that is $q$, is activated during each symbol time). Every column of the selected matrix is then transmitted from the $M$-antenna array at a baud-rate $T / T_{B}$, where $T_{B}$ is the time duration of the transmitted block. 
Four parameters characterize STSK transmission: the number of transmit antennas $M$, the number of receive antennas $N$, the cardinality of the set of dispersion matrices $Q$ and, finally, $T$. Specific STSK configurations are then identified by a vector of 4 parameters: $(M, N, T, Q)$, where $T$ actually accounts the degree of time diversity provided by STSK coding.

The DM sets are generated offline using Genetic Algorithm (GA)-based optimization [13]. The generation process is beyond the scope of this paper and is not discussed here for sake of brevity. However, we encourage interested readers to read more about generation of GA-aided dispersion matrix in [13]. At the receiver side, the signal is first frequencydownconverted. After A/D conversion and filtering, the cyclic prefix is removed from the digital CP-SC-STSK signal. Then, the sample stream is serial-to-parallel $(\mathrm{S} / \mathrm{P})$ converted and transformed in the frequency domain by a $B$-point FastFourier Transform (FFT). It is assumed that channel remains stationary over a block duration of $T_{B}$ [9]. Under this assumption, the received and FFT transformed SC-STSK signal matrix of $B N \times T$ size, denoted by $\mathbf{Y}_{\mathrm{STSK}}$, can be expressed as follows:

$$
\mathbf{Y}_{\mathrm{STSK}}=\mathbf{H S}_{\mathrm{STSK}}+\mathbf{Z}
$$

where $\mathbf{H}$ is the frequency-domain mm-Wave channel matrix of size $B N \times B M, \mathbf{Z}$ is the $B N \times T$ received AWGN vector with zero-mean and $\sigma^{2}$ variance i.i.d. components, while $\mathbf{S}_{\mathrm{sTSK}}=$ $\left[\mathbf{X}_{\text {STSK }}(1), \cdots, \mathbf{X}_{\text {STSK }}(B)\right]^{\prime}$ is the block of transmitted STSK codewords of size $B M \times T$. The reduced complexity suboptimum MMSE-FDE receiver architecture of [11], orginally considered for OFDMA/SCFDMA, is employed also for the proposed CP-SC-STSK system. Zero Forcing (ZF) FDE is not advisable, as it enhances noise at the output of demodulation process which degrades the link performance. The MMSE filter-weight matrix $W$ is given as,

$$
W_{\text {MMSE }}^{\mathrm{H}}=\left[H^{\mathrm{H}} H+\sigma^{2} \mathbf{I d}_{\mathrm{BM}}\right]^{-1} H^{\mathrm{H}} \in \mathbb{C}^{B M \times B N}
$$

where $(\cdot)^{\mathrm{H}}$ is the Hermitian operation. For the sake of simplicity, we omit MMSE subscripts from the equations hereafter. The equalized STSK block $\bar{V}_{\text {STSK }} \in \mathbb{C}^{B M \times T}$, is given as,

$$
\bar{V}_{\text {STSK }}=W^{\mathrm{H}} \mathbf{Y}_{\text {STSK }}=\bar{S}_{\text {STSK }}+\bar{Z}
$$

where $\bar{S}_{\text {STSK }}$ is the equalized codeword block and $\bar{Z}$ is Gaussian noise matrix made of zero-mean i.i.d elements of variance $\|W\|^{2} \sigma^{2}$. To ease the computations, vectorial stacking, $\operatorname{vec}(\cdot)$, is applied on the STSK received codeword $\bar{\Psi}_{\text {STSK }}$, obtained by the parallel-to-serial (P/S) conversion of $\bar{V}_{\text {STSK }}$, as in [9],

$$
\widetilde{\Psi}_{\text {STSK }}=\operatorname{vec}\left(\bar{\Psi}_{\text {STSK }}\right) \in \mathbb{C}^{M T \times 1}
$$

Finally, the space-time demapping operation in Fig. 1 is performed by means of a joint estimation of selected DM index $\hat{q}$ and K-ary QAM/PSK symbol $\hat{S}$ as given in the following:

$$
(\hat{q}, \hat{S})=\underset{q, x}{\arg \min }\left\|\hat{\Psi}_{\mathrm{STSK}}-\boldsymbol{\kappa}_{q, k}\right\|^{2}
$$

where:

$$
\hat{\Psi}_{\text {STSK }}=\frac{1}{T} \mathbf{A}^{\mathrm{H}} \widetilde{\Psi}_{\text {STSK }}=\left[v_{1}, \ldots, v_{Q}\right] \in \mathbb{C}^{Q \times 1}
$$

and:

$$
\boldsymbol{\kappa}_{q, k}=\mathbf{A} \chi_{q, k}
$$

A being the DM set as in (10) and $\chi_{q, k}$ is the $k$-th symbol from $K$-QAM/PSK signal set located at $q$-th position in the vector given as in (11),

$$
\begin{gathered}
\mathbf{A}=\left[\operatorname{vec}\left(A_{1}\right), \operatorname{vec}\left(A_{2}\right), \cdots, \operatorname{vec}\left(A_{Q}\right)\right] \\
\chi_{q, k}=[\underbrace{0, \cdots, 0}_{q-1}, S_{k}, \underbrace{0, \cdots, 0}_{Q-q}]
\end{gathered}
$$

As far as, the computational complexity of the sub-optimal CP-SC-STSK receiver with MMSE is concerned, the number of elementary operations required to detect an STSK symbol is: $O\left(4 M^{2} N+8 M N+4 M T Q+2 Q K^{\prime}+Q+2 K\right)$ [11], where $K^{\prime}$ is the number of symbols lying in the first quadrant of the constellation.

The spectral efficiency per symbol of CP-SC-STSK, denoted by $\eta$, can be expressed as,

$$
\eta=\frac{\log _{2}(K)+\log _{2}(Q)}{T(1+\alpha)}[b / s / H z]
$$

where $K$ is the number of symbols of the K-ary PSK or QAM constellation and $\alpha$ the roll-off factor of the raised cosine pulse shaping. From (12), it is easy to understand that the throughput of STSK does not depend on the number of antenna elements and, therefore, on RF hardware complexity. Fixing $K$ and $\alpha$, the value of $\eta$ increases with the the cardinality of the dispersion matrix $Q$ and decreases with the STSK coding factor $T$. Large values of $Q$ are not advisable, as the pairwise error probability involving STSK codeword would increase too much with a consequentially severe link performance degradation [9]. On the other hand, an increase of $T$ would be theoretically beneficial for link performance, because the anti-multipath diversity of STSK is quantified by the formula: $\operatorname{Nmin}(M, T)$ [9]. The setting of parameters $Q$ and $T$ is therefore crucial in determining the multiplexing gain and the diversity performance of STSK.

\section{B. The state-of-the-art SM-aided Cyclic-Prefixed Single Car- rier Block Transmission}

The proposed CP-SC-STSK is compared with CP-SC-SM scheme shown in [8]. CP-SC-SM activates only a single $m$ th antenna out of available $M$ transmit antennas for each KQAM/PSK symbol $x$ of the $B$-length CP-SC block $\mathbf{S}_{\mathrm{SM}}$ (see Fig. 2). The TX/RX chain is rather similar to that described in subsection II-A, except for the spatially-multiplexed transmission (that can be regarded as a special case of STSK [14]) and space-time demapping that is performed on the basis of spatial de-multiplexing described in the following.

First of all, let's denote $\bar{V}_{\mathrm{SM}} \in \mathbb{C}^{B M \times 1}$ the SC-SM block after $\mathrm{CP}$ removal and frequency-domain equalization. It can be expressed as,

$$
\bar{V}_{\mathrm{SM}}=\bar{S}_{\mathrm{SM}}+\bar{\zeta}
$$

$\bar{S}_{\mathrm{SM}}$ being the equalized SM block and $\bar{\zeta}$ the Gaussian noise matrix coming out from equalization filter. Then, antenna 

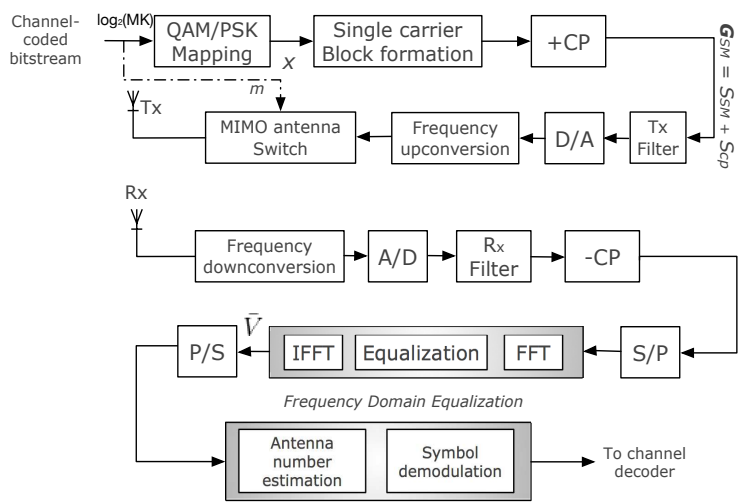

Fig. 2. Block diagram of the state-of-the-art Spatial Modulation (SM)-aided Cyclic-Prefixed Single-Carrier Transceiver System (CPSC-SM) [8].

number estimation $\hat{m}$ and symbol demodulation $\hat{x}$ are given in (14) and (15), respectively,

$$
\begin{aligned}
& (\hat{m}, \hat{x})=\min _{m, x}\left\|\bar{\Psi}_{S M}-\gamma(m, x)\right\|^{2} \\
& \gamma(m, x)=[\underbrace{0, \cdots, 0}_{m-1}, x, \underbrace{0 \cdots, 0}_{M-m}]
\end{aligned}
$$

where $\bar{\Psi}_{S M} \in \mathbb{C}^{M \times 1}$ is the received SM codeword obtained by the P/S conversion of $\bar{V}_{\mathrm{SM}}$. The computational complexity of the sub-optimum MMSE-FDE receiver for CP-SC-SM is given as $O\left(4 M^{2} N+8 M N+2 K\right)$ that is slightly reduced with respect to that of CP-SC-STSK. Now, it is interesting to analyse the spectral efficiency per symbol achievable by CP-SC-SM, denoted by $\eta^{\prime}$ that is given as,

$$
\eta^{\prime}=\frac{\log _{2}(K)+\log _{2}(M)}{(1+\alpha)}[b / s / H z]
$$

Fixing, as usual, the values of $K$ and $\alpha$, the comparison of spectral efficiency is generally in favor of CP-SC-SM. Indeed, in CP-SC-STSK the parameter $T$ is a positive integer always higher than 1 in order to provide transmit diversity (if $T=1$, STSK somewhat degenerates in SM, as shown in [14]) and the parameter $Q$ is kept moderately low in order to guarantee satisfactory link performance. Therefore, $\eta^{\prime} \geqslant \eta$ in most of the cases. It is expected that the potential improvement of link performance yielded by CP-SC-STSK will be paid in terms of throughput reduction. These issues will be better detailed in section IV.

\section{LINK IMPAIRMENT ANALYSIS}

\section{A. Mm-wave multipath channel modeling}

The Statistical Spatial Channel Model (SSCM) used in this work is based on temporal clusters and spatial lobes to model mmWave channel [15]. Temporal clusters are composed of many intra-cluster subpaths with different random delays. As in [15] the directional Power Density Profile (PDP) of mmwave SSCM is given as,

$$
\begin{array}{r}
h\left(t, \vec{\Theta}_{d}, \vec{\Phi}_{d}\right)=\sum_{p=1}^{P_{t}} \sum_{u=1}^{U_{p}} a_{u, p} e^{j \varphi_{u, p}} \varrho\left(t-\tau_{u, p}\right) . \\
g_{T X}\left(\vec{\Theta}_{d}-\vec{\Theta}_{u, p}\right) \cdot g_{R X}\left(\vec{\Theta}_{d}-\vec{\Theta}_{u, p}\right)
\end{array}
$$

where, $t=$ absolute propagation time, $\vec{\Theta}_{d}=(\theta, \phi)_{T X}$ and $(\theta, \phi)_{R X}=$ azimuth/elevation Angle of Departure (AoD) and Angle of Arrival (AoA), respectively. $\left(P_{t}, U_{p}\right)$ represent the number of time clusters and number of cluster subpaths, respectively, $a_{u, p}$ is the amplitude of $u$-th subpath in $p$-th time cluster, $\left(\varphi_{u, p}, \tau_{u, p}\right)$ are the phase and propagation time delays, respectively. $g_{T X}(\vec{\Phi})$ and $g_{R X}(\vec{\Phi})$ are the arbitrary 3-D (azimuth and elevation) Tx and Rx complex amplitude MIMO antenna patterns. The non-parametric omnidirectional MIMO channel $\mathbf{H}_{l}$ for $l$-th multipath component is given as,

$$
\mathbf{H}_{l}=\mathbf{R}_{n}^{1 / 2} \mathbf{H}_{w} \mathbf{R}_{m}^{1 / 2}
$$

where $\mathbf{H}_{l}$ is $N \times M$ MIMO channel matrix. $\mathbf{R}_{n}$ and $\mathbf{R}_{m}$ are receive and transmit spatial correlation matrices, respectively. $\mathbf{H}_{w}$ is the matrix of small scale spatial path amplitudes and phases as in (17). $\mathbf{H}_{l}$ retains the characteristics of autocorrelation of multipath components specified through spatial correlation matrices as in (18) while retaining the small scale distribution specified in $\mathbf{H}_{w}$ (Rician in case of LoS and Rayleigh in case of $\mathrm{nLoS}$ ).

\section{B. Impact of phase noise}

Non-ideal oscillators give rise to Phase Noise (PN) in hardware transceivers especially at $\mathrm{mm}$-Wave band. This motivates system engineers to equip hardware with low PN high frequency oscillators to minimize frequency and phase drifts [16]. Wide-band systems require front-end components to operate at higher bandwidths resulting in increasing the phase perturbation introduced by entire communication system. Hence the question, "How much phase noise a receiver can tolerate without compromising system performance?", deserves an answer. In this paper, we try to answer this question by shedding light on both considered systems, CPSC-STSK and CP-SC-SM, in the presence of noisy oscillators. For the sake of simplicity, we drop the subscripts (STSK and SM) for better readers' understanding.

The equalized codeword symbol $\bar{V}_{\mathrm{X}}$ in the presence of phase noise can be expressed as follows,

$$
\bar{V}=\bar{X} \sum_{n=0}^{N-1} \mathbf{c}_{n}+\bar{Z}
$$

where $\mathbf{c}$ of size $M \times T$ is the gain matrix obtained as a result of equalization operation that increases the output signal to noise ratio (SNR) of each codeword. In the presence of PN in the system, (19) can be written as,

$$
\bar{V}=\bar{X} \sum_{n=0}^{N-1} \mathbf{c}_{n} e^{j \theta_{n}^{\mathrm{x}}}+\bar{Z}
$$


$e^{j \theta_{n}^{\mathrm{x}}}$ is phase noise on codeword symbol $X$, where $\theta_{n}$ is the Gaussian random noise with PSD as specified by the receiver oscillator [17]. Usually phase noise variance is very low (i.e., $\theta_{n}<<1$ ), we can approximate the phase noise exponential as $e^{j \theta_{n}} \approx 1+j \theta_{n}$. By expanding the multiplicative term in (20), we get:

$$
\begin{array}{r}
\sum_{n=0}^{N-1} \mathbf{c}_{n}\left(1+j \theta_{n}\right)=\mathbf{c}_{0}+\mathbf{c}_{1}+\cdots+\mathbf{c}_{N-1}+ \\
\underbrace{j \mathbf{c}_{0} \theta_{0}+j \mathbf{c}_{1} \theta_{1}+\cdots+j \mathbf{c}_{N-1} \theta_{N-1}}_{\approx 0}
\end{array}
$$

With $N \rightarrow \infty$, we are left with space-time diversity terms $\mathbf{c}_{n}$ in (21). STSK system acts against phase noise by combining the received energy over space and time. Increasing $T$ such that $M \leq T$ will further improve the performance against PN. Considering CP-SC-SM system, we have $\mathbf{c}$ of size $M \times 1$, gain matrix from (19), with just single transmitting antenna. Indeed, with $N$ sufficiently large the diversity terms will dominate over phase noise terms in (21). However in case of high phase noise in the system, SM performance will degrade for small MIMO systems (i.e., upto 4 antennas). In STSK, spacetime structure averages the phase noise, while only receiver diversity averages out the phase noise effect in SM due to the fact that $\theta_{n}$ is Gaussian as in (21).

\section{Impact of channel estimation error}

The effectiveness of the FDE operation strictly depends on the accuracy of the knowledge of frequency-domain Channel State Information (CSI), accounted in our system by the matrix $H$. A bad estimation of $H$ would involve imperfect equalization and, therefore, results into inter-symbol interference.

Channel estimation in single carrier systems is a critical issue in practical highly fluctuating environments (i.e., $\mathrm{nLoS}$ channels). Usually at the receiver side, an efficient channel estimator is required to perform coherent detection. However pilot-signal overhead increases in rapidly varying channels resulting in reduced system throughput.

We analyse the performance of STSK and SM systems in the presence of channel estimation error wherein the channel estimation error is modelled with the help of zero-mean complex random gaussian noise $\varepsilon$ of variance $\sigma_{e}^{2}$ added to actual CSI between any $m$-th $T_{x}$ and $n$-th $R_{x}$ pair as in [18],

$$
\hat{H}_{\mathrm{m}, \mathrm{n}}=H_{\mathrm{m}, \mathrm{n}}+\varepsilon
$$

For example, an arbitrary $\alpha$ codeword, $M=2$ and $T=2$, is given as,

$$
\alpha=\left[\begin{array}{ll}
a_{1} & a_{2} \\
a_{3} & a_{4}
\end{array}\right]
$$

After equalizing (23), we have equalized codeword $\alpha$ of size $(M \times T)$,

$$
\left[\begin{array}{ll}
a_{1}\left(\left|c_{1}\right|^{2}+\left|c_{2}\right|^{2}\right)+a_{1}\left(c_{1}+c_{2}\right) \varepsilon & a_{2}\left(\left|c_{1}\right|^{2}+\left|c_{2}\right|^{2}\right)+a_{2}\left(c_{1}+c_{2}\right) \varepsilon \\
a_{3}\left(\left|c_{1}\right|^{2}+\left|c_{2}\right|^{2}\right)+a_{3}\left(c_{1}+c_{2}\right) \varepsilon & a_{4}\left(\left|c_{1}\right|^{2}+\left|c_{2}\right|^{2}\right)+a_{4}\left(c_{1}+c_{2}\right) \varepsilon
\end{array}\right]
$$

where, $c_{1}=H_{1,1}$ and $c_{2}=H_{1,2}$. For $\sigma_{e}^{2}<<1$, STSK with space-time diversity helps in mitigating the error term $\varepsilon$ in demodulation process as in (24) where diversity overshadows the errors in channel estimation. However in case of high $\sigma_{e}^{2}$, we have dominant error term in (24) reducing the diversity gain and hence making coherent demodulation infeasible. In case of SM, the equalized matrix at the input of demodulation process is,

$$
\left[\begin{array}{l}
\bar{X}\left(\left(\left|c_{1}\right|^{2}+\left|c_{2}\right|^{2}\right)+\left(c_{1}+c_{2}\right) \varepsilon\right) \\
\bar{X}\left(\left(\left|c_{1}\right|^{2}+\left|c_{2}\right|^{2}\right)+\left(c_{1}+c_{2}\right) \varepsilon\right)
\end{array}\right]
$$

Unlike in STSK (24) where we have space-time diversity providing an edge over imperfect channel estimation, we see in (25) that SM only relies on receiver diversity to counter imperfect channel estimation impairment. Thus, the degradation due to channel errors is noticeable for extreme channel conditions for SM MIMO systems and when $\varepsilon$ is high. Such analysis shows that SM performance will be compromised for high mobile broadband users.

\section{Simulation RESUlts}

Two series of MATLAB simulation results will be discussed in this section. The first series of results is targeted at assessing phase noise effects, whereas the second series of results will consider imperfect channel estimation. Both series will provide results achieved in Line-of-Sight (LoS) and nonLine-of-Sight (nLoS) channel conditions. The proposed CPSC-STSK technique will be compared with CP-SC-SM. The parameters of MIMO coding and modulation are listed in Table I, while simulation parameters are reported in Table II. Convolutional coding with random interleaving is considered with code-rate $1 / 2$ in both schemes, where this low code-rate ensures robustness against noise and channel impairments.

Considering the parameters of Tab. I and II, together with

TABLE I

MIMO SYSTEM PARAMETERS

\begin{tabular}{|c|c|c|c|}
\hline \multicolumn{2}{|c|}{ Space-Time Shift Keying (STSK) } & \multicolumn{2}{|c|}{ Spatial Modulation (SM) } \\
\hline $2 \times 2$ MIMO & $4 \times 4$ MIMO & $2 \times 2$ MIMO & $4 \times 4$ MIMO \\
\hline$K=4$ (QPSK) & $K=4$ (QPSK) & $K=2($ BPSK) & $K=4$ (QPSK) \\
\hline$Q=4, T=2$ & $Q=4, T=2$ & $M=2$ & $M=4$ \\
\hline
\end{tabular}

TABLE II

SIMULATION PARAMETERS

\begin{tabular}{|l|l|}
\hline Parameters & Values \\
\hline Bandwidth & $500 \mathrm{MHz}$ \\
\hline Frequency Band & $73 \mathrm{Ghz}$ \\
\hline Block size, $B$ & 512 \\
\hline Cyclic Prefix, $S_{c p}$ (samples) & 150 \\
\hline Pulse shaping filter & Root Raised Cosine (roll-off 0.35) \\
\hline
\end{tabular}

the spectral efficiency in (12) and (16), we can immediately compute the pure data bitrate (net of channel coding and cyclic prefix) provided by the different assessed cyclic-prefixed single-carrier MIMO techniques, namely: $286.44 \mathrm{Mb} / \mathrm{s}$ for $(2,2,2,4)$ and $(4,4,2,4)$ STSK, $286.44 \mathrm{Mb} / \mathrm{s}$ and $572.89 \mathrm{Mb} / \mathrm{s}$ for $2 \times 2 \mathrm{SM}$ and $4 \times 4 \mathrm{SM}$, respectively. In the $4 \times 4 \mathrm{MIMO}$ case, SM provides a doubled transmission throughput with respect to $(4,4,2,4)$ STSK. These numbers confirm what we 


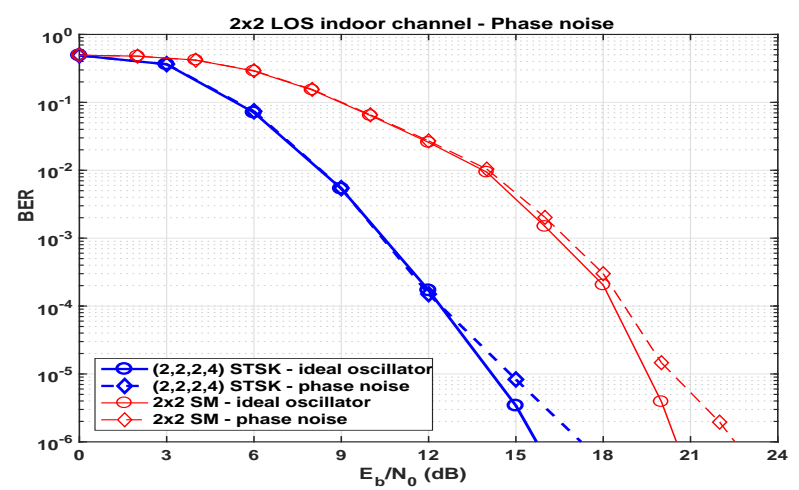

Fig. 3. BER Performance of $(2,2,2,4)$ CP-SC-STSK vs. $2 \times 2$ CP-SC$\mathrm{SM}$ in the presence of the phase noise profile: indoor LoS channel with Rice factor $\rho_{d B}=10$.

stated in subsection II-B about the tradeoff between throughput and transmit diversity inherent to STSK. Simulation results will show in the following subsections, if and when such a price is conveniently paid to obtain a substantial link performance improvement.

1) Phase Noise analysis: The phase noise profile used for our simulations has power spectral density (PSD) given as $\left[\begin{array}{llll}-90 & -101 & -111 & -132\end{array}\right](\mathrm{dBc} / \mathrm{Hz})$ at frequency offsets $\left[\begin{array}{lll}1 & 5 & 10\end{array}\right.$ $100](\mathrm{MHz})$. It is related to a frequency synthesizer providing unmodulated carriers in the range of $73.9-83.5 \mathrm{GHz}$ [17]. The multipath channel has been parameterized in different manner as far as indoor or outdoor environment is considered. In particular, nLoS propagation and higher number of path clusters have been considered for the outdoor channel. On the other hand, LoS propagation (Rice factor set to $10 \mathrm{~dB}$ ) and lower fading clusterization have been considered for indoor channel.

For the $2 \times 2$ MIMO system, CP-SC-STSK attains gain up to $4 \mathrm{~dB}$ over CP-SC-SM as shown in Fig. 3, when the oscillator is ideal. In case of using the real mm-Wave oscillator with considered phase noise profile, CP-SC-STSK technique, thanks to augmented space-time diversity, is more resilient to phase noise and, hence, able at preserving signal after demodulation, as already stated in Section III. Especially in LoS conditions, PN does not affect too much CP-SC-STSK and $\mathrm{CP}-\mathrm{SC}-\mathrm{SM}$, as in Fig. 3. However in nLoS conditions, small-scale MIMO systems experience significant degradation, where an irreducible error floor is noticed for CP-SC-SM and slow BER-decay experienced by CP-SC-STSK in Fig. 4. Anyway, in this last case, the performance improvement yielded by STSK-based transmission is dramatic. Such destructive phase noise impact can be contrasted with the help of larger-scale MIMO. (4,4,2,4)-QPSK and 4×4-QPSK SM perform remarkably against phase noise both in LoS and $\mathrm{nLoS}$ conditions, as shown in Figs. 5-6. This is primarily due to increased receiver diversity helping against phase noise. In general, 4-antenna STSK system outperforms the 4-antenna SM system in both channel scenarios under the presence of

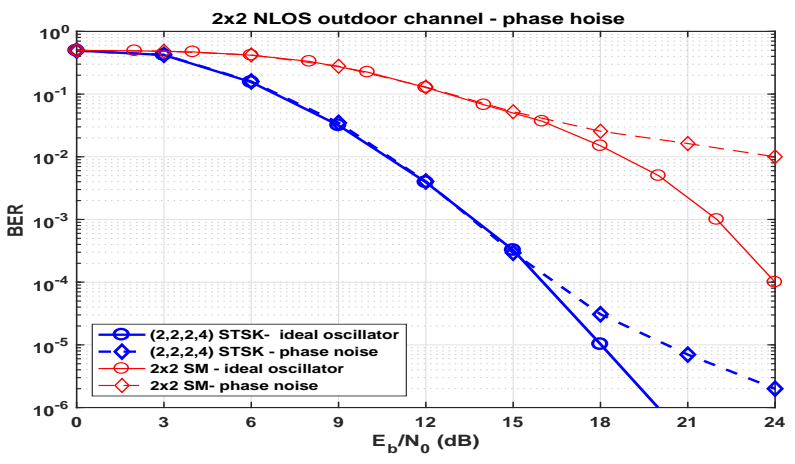

Fig. 4. BER Performance of $(2,2,2,4)$ CP-SC-STSK vs. $2 \times 2$ CP-SC$\mathrm{SM}$ in the presence of the phase noise profile: outdoor $\mathrm{nLoS}$ channel.

phase noise. It should be highlighted in Fig. 6 the $12 \mathrm{~dB}$ performance improvement attained by $(4,4,2,4)$ CP-SC-STSK against $4 \times 4 \mathrm{CP}-\mathrm{SC}-\mathrm{SM}$ under $\mathrm{nLoS}$ outdoor conditions. In such a case, the augmented power efficiency clearly overcomes the 3dB-reduced spectral efficiency.

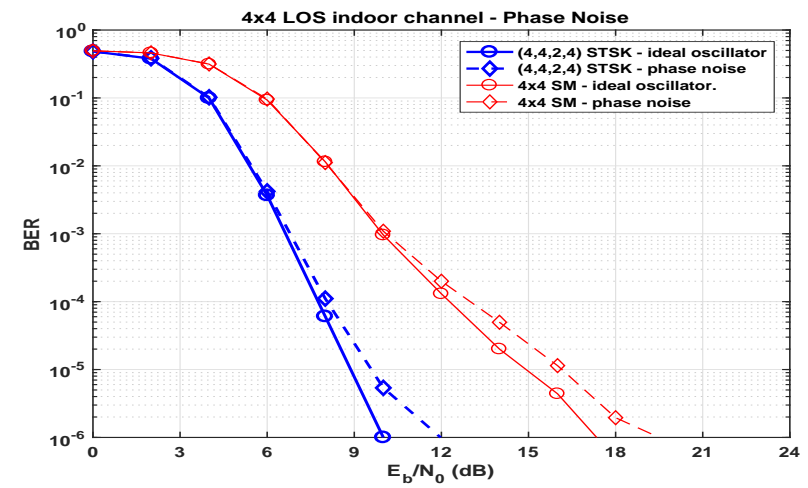

Fig. 5. BER Performance of $(4,4,2,4)$ CP-SC-STSK vs. $4 \times 4$ CP-SC$\mathrm{SM}$ in the presence of the phase noise profile: indoor LoS channel with Rice factor $\rho_{d B}=10$.

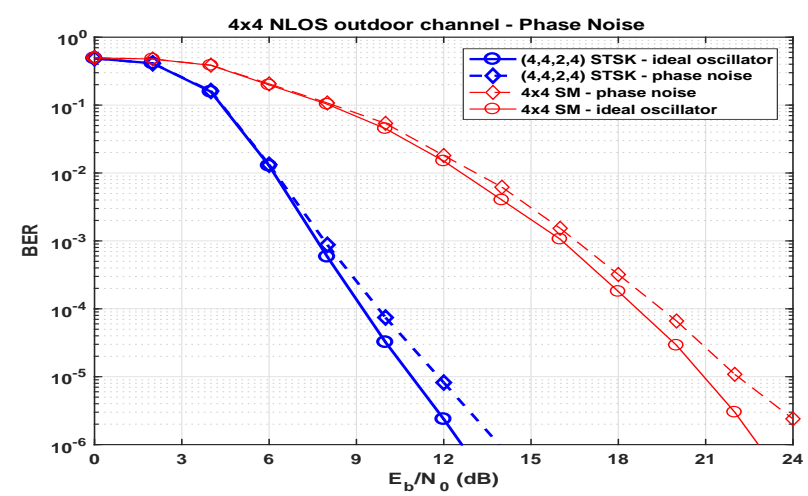

Fig. 6. BER Performance of $(4,4,2,4)$ CP-SC-STSK vs. $4 \times 4$ CP-SC$\mathrm{SM}$ in the presence of the phase noise profile: outdoor nLoS channel. 
2) Channel Esimation Analysis: We try to analyse the performance of the compared CP-SC-STSK and CP-SC-SM in the presence of channel estimation errors, as described in Section III. We model the imperfect channel estimation as in (22).

Let's consider first, the $2 \times 2$ MIMO configuration. With estimation error variance $\sigma_{e}^{2}=-30 \mathrm{~dB}$, a small degradation of $1 \mathrm{~dB}$ is noticed for $(2,2,2,4)$ CP-SC-STSK in indoor LoS scenario as compared to larger $2.5 \mathrm{~dB}$ degradation measured for CP-SC-SM system as shown in Fig. 7. It should be noticed that for $\sigma_{e}^{2}=-30 \mathrm{~dB}$, the performance degradation of all the assessed techniques, although not negligible, does not exceed $3 \mathrm{~dB}$ in both the considered transmission scenarios (see Figs. 7 and 8). On the other hand, $(2,2,2,4)$ CP-SC-STSK and $2 \times 2 \mathrm{CP}-\mathrm{SC}-\mathrm{SM}$ transmissions fail considerably in case of $\sigma_{e}^{2}=-10 d B$, as all related BER curves of Figs. 7 and 8 exhibit an evident error floor. An important observation: in $(2,2,2,4)$ where the high channel estimation error results into negative diversity in high-SNR regime due to the fact the space-time decoding, terms involving $\varepsilon$ negatively adds up in (24). Apparently, such a channel estimation error neutralizes the diversity inherent to MIMO transmission as seen in Section III-C.

With 4-antenna configuration, CP-SC-STSK has shown a

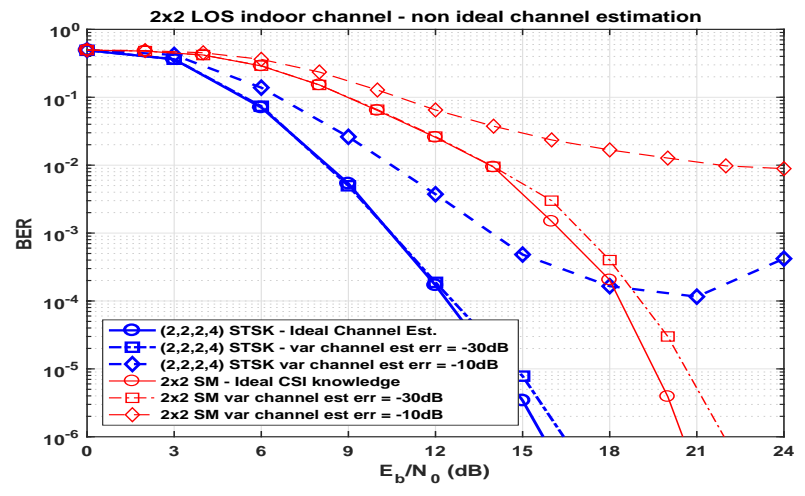

Fig. 7. BER Performance of $(2,2,2,4)$ CP-SC-STSK vs. $2 \times 2 \mathrm{CP}$ $\mathrm{SC}-\mathrm{SM}$ in the presence of non-ideal channel estimation: indoor LoS channel with Rice factor $\rho_{d B}=10$.

remarkable performance improvement in case of non-ideal CSI knowledge, both with lower and higher channel estimation error variance. Having a maximum performance degradation of $2 \mathrm{~dB}$ in the LoS scenario and $3 \mathrm{~dB}$ in the $\mathrm{nLoS}$ scenario for $\sigma_{e}^{2}=-10 d B$, STSK outpeforms SM that is more prone to highly inaccurate channel estimation even when employed with larger array size, as cleary shown by the BER curves of Fig.9 and 10. When the channel estimation accuracy improves $\left(\sigma_{e}^{2}=-30 d B\right)$, the performance degradation measured for CP-SC-SM becomes more acceptable both under LoS and nLoS propagation conditions.

3) Computational Complexity: The computational complexities associated with configurations specified in Tab. I for cyclic-prefixed single carrier STSK and SM are reported in Tab. III. The computational complexity is specified in terms

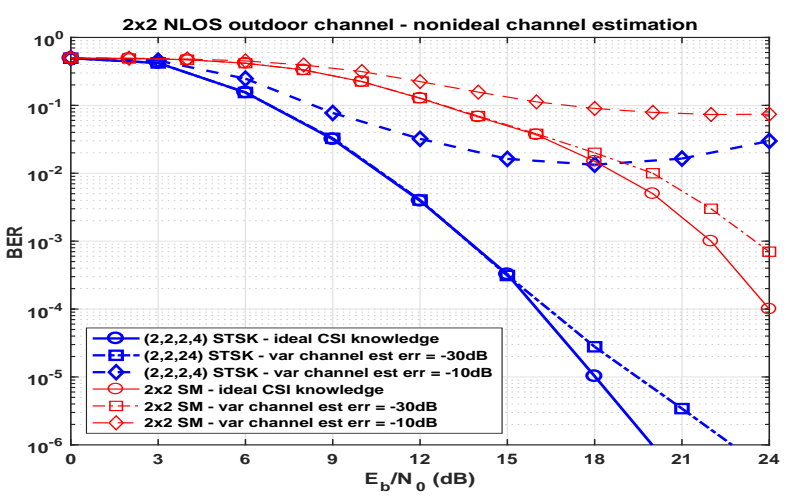

Fig. 8. BER Performance of $(2,2,2,4)$ CP-SC-STSK vs. $2 \times 2$ CP-SC$\mathrm{SM}$ in the presence of non-ideal channel estimation: outdoor $\mathrm{nLoS}$ channel.

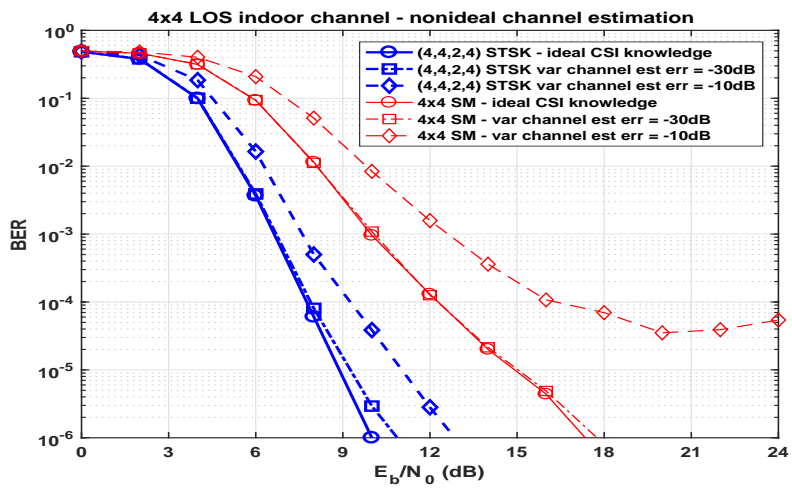

Fig. 9. BER Performance of $(4,4,2,4)$ CP-SC-STSK vs. $4 \times 4$ CP$\mathrm{SC}-\mathrm{SM}$ in the presence of non-ideal channel estimation: indoor LoS channel with Rice factor $\rho_{d B}=10$.

of elementary operations per single carrier block given in third column of Tab. III. The lowest computational complexity is for $2 \times 2 \mathrm{CP}-\mathrm{SC}-\mathrm{SM}$ with a highly-compromised BER performance as noticed in earlier subsections. The computational complexity of $(4,4,2,4)$ CP-SC-STSK is highest amongst all considered MIMO techniques, but it is still affordable with excellent BER performance measured in all the assessed transmission scenarios.

\section{CONClusion}

In this paper we analyse the performance of cyclic-prefixed single carrier STSK scheme for broadband indoor and outdoor networking applications in mm-Wave environments. In order to have complete picture of the analyses, we test the robustness of the proposed CP-SC-STSK systems against phase noise and non-ideal channel estimation, in LoS indoor and $\mathrm{nLoS}$ outdoor propagation scenarios. Thanks to the augmented diversity, attained in space-time and frequency domain, CP-SC-STSK, as compare to state-of-the-art CP-SC-SM, has shown significant resilience against phase noise characterizing $\mathrm{mm}$-Wave oscillators, in particular under critical nLoS outdoor propagation conditions. As far as non-ideal channel estimation is 
TABLE III

COMPUTATIONAL COMPLEXITY

\begin{tabular}{|l|c|l|}
\hline \multicolumn{1}{|c|}{ MIMO Technique } & Order of computational complexity & $\begin{array}{l}\text { \# OF ELEMENTARY OPERATIONS } \\
\text { PER Single Carrier Block SYMBOL }\end{array}$ \\
\hline Proposed Single Carrier STSK & $\left(4 M^{2} N+8 M N+4 M T Q+2 Q K^{\prime}+Q+2 K\right)$ & $1.48 \times 10^{2}$ for $(2,2,2,4)-$ QPSK \\
\cline { 3 - 3 } & & $5.32 \times 10^{2}$ for $(4,4,2,4)-$ QPSK \\
\cline { 3 - 3 } Single Carrier SM [8] & $\left(4 M^{2} N+8 M N+2 K\right)$ & 68 for $2 \times 2-$ BPSK \\
\cline { 3 - 3 } & & $3.92 \times 10^{2}$ for $4 \times 4$ - QPSK \\
\hline
\end{tabular}

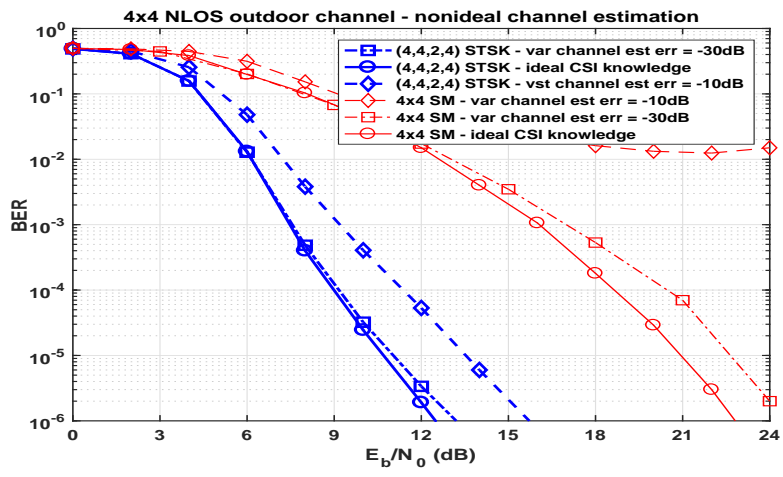

Fig. 10. BER Performance of $(4,4,2,4)$ CP-SC-STSK vs. $4 \times 4$ CP-SC$\mathrm{SM}$ in the presence of non-ideal channel estimation: outdoor $\mathrm{nLoS}$ channel.

concerned, CP-SC-STSK with larger antenna array has shown remarkable performance also in presence of high estimation error variance, while CP-SC-SM always suffers a lot from nonprecise knowledge of channel state information. The price to be paid by CP-SC-STSK is in terms of throughput reduction (halved in the $4 \times 4$ MIMO case) and slightly increased receiver complexity.

\section{REFERENCES}

[1] M. Coldrey, J.-E. Berg, L. Manholm, C. Larsson, and J. Hansryd, "Nonline-of-sight small cell backhauling using microwave technology," IEEE Communications Magazine, vol. 51, pp. 78-84, September 2013.

[2] M. R. Akdeniz, Y. Liu, M. K. Samimi, S. Sun, S. Rangan, T. S. Rappaport, and E. Erkip, "Millimeter Wave Channel Modeling and Cellular Capacity Evaluation," IEEE Journal on Selected Areas in Communications, vol. 32, pp. 1164-1179, June 2014.

[3] "IEEE Standard for Information technology-Telecommunications and information exchange between systems-Local and metropolitan area networks-Specific requirements-Part 11: Wireless LAN Medium Access Control (MAC) and Physical Layer (PHY) Specifications Amendment 3: Enhancements for Very High Throughput in the $60 \mathrm{GHz}$ Band," IEEE Std 802.11ad-2012 (Amendment to IEEE Std 802.11-2012, as amended by IEEE Std 802.11ae-2012 and IEEE Std 802.11aa-2012), pp. 1-628, Dec 2012.

[4] Z. Wang, X. Ma, and G. Giannakis, "OFDM or single-carrier block transmissions?," IEEE Transactions on Communications, vol. 52, pp. 380-394, March 2004.

[5] Y. Toriyama, K. Kojima, T. Taniguchi, M. Zhang, and J. Hirokawa, "Multi-level QAM single-carrier high-efficiency broadband wireless system for millimeter-wave applications," in 2010 IEEE Radio and Wireless Symposium (RWS), pp. 677-680, Jan 2010.

[6] J. C. Silva, R. Dinis, N. Souto, and M. M. da Silva, "MIMO SCFDE Transmission Techniques with Channel Estimation and High-order Modulations," Session 1A3b MIMO Systems, p. 47, 2013.

[7] R. Y. Mesleh, H. Haas, S. Sinanovic, C. W. Ahn, and S. Yun, "Spatial Modulation," IEEE Transactions on Vehicular Technology, vol. 57, pp. 2228-2241, July 2008.
[8] R. Rajashekar, K. Hari, and L. Hanzo, "Spatial Modulation Aided ZeroPadded Single Carrier Transmission for Dispersive Channels," IEEE Transactions on Communications, vol. 61, pp. 2318-2329, June 2013.

[9] S. Sugiura, S. Chen, and L. Hanzo, "Coherent and differential spacetime shift keying: A dispersion matrix approach," IEEE Transactions on Communications, vol. 58, pp. 3219-3230, November 2010.

[10] C. Sacchi, T. Rahman, I. A. Hemadeh, and M. El-Hajjar, "Millimeterwave transmission for small-cell backhaul in dense urban environment: a solution based on mimo-ofdm and space-time shift keying (stsk)," IEEE Access, vol. PP, no. 99, pp. 1-1, 2017.

[11] M. Kadir, S. Sugiura, J. Zhang, S. Chen, and L. Hanzo, "OFDMA/SCFDMA aided space time shift keying for dispersive multiuser scenarios," IEEE Transactions on Vehicular Technology, vol. 62, pp. 408-414, Jan 2013.

[12] J. Wu and S. D. Blostein, "Linear Dispersion for Single-Carrier Communications in Frequency Selective Channels," in IEEE Vehicular Technology Conference, pp. 1-5, Sept 2006.

[13] F. Babich, A. Crismani, M. Driusso, and L. Hanzo, "Design Criteria and Genetic Algorithm Aided Optimization of Three-Stage-Concatenated Space-Time Shift Keying Systems," IEEE Signal Processing Letters, vol. 19, pp. 543-546, Aug 2012.

[14] M. I. Kadir, S. Sugiura, S. Chen, and L. Hanzo, "Unified MIMOMulticarrier Designs: A Space-Time Shift Keying Approach," IEEE Communications Surveys Tutorials, vol. 17, pp. 550-579, Secondquarter 2015.

[15] M. K. Samimi and T. S. Rappaport, "3-D statistical channel model for millimeter-wave outdoor mobile broadband communications," CoRR, vol. abs/1503.05619, 2015.

[16] H. Mehrpouyan, M. Khanzadi, M. Matthaiou, A. Sayeed, R. Schober, and Y. Hua, "Improving bandwidth efficiency in E-band communication systems," IEEE Communications Magazine, vol. 52, pp. 121-128, March 2014.

[17] J. O. Plouchart, M. Ferriss, B. Sadhu, M. Sanduleanu, B. Parker, and S. Reynolds, "A 73.9 to $83.5 \mathrm{GHz}$ synthesizer with $111 \mathrm{dBc} / \mathrm{Hz}$ phase noise at $10 \mathrm{MHz}$ offset in a $130 \mathrm{~nm}$ SiGe BiCMOS technology," in 2013 IEEE Radio Frequency Integrated Circuits Symposium (RFIC), pp. 123126, June 2013.

[18] C. H. Yih, "Effects of Channel Estimation Error on the BER Performance of OFDM Systems in Multipath Rayleigh Fading Channels," in 2007 IEEE 66th Vehicular Technology Conference, pp. 1097-1101, Sept 2007. 